\title{
Inhibitors of sodium-glucose transport protein 2: A new multidirectional therapeutic option for heart failure patients
}

\author{
Jacek Kubica $^{1}$, Aldona Kubica ${ }^{1}$, Klaudyna Grzelakowska ${ }^{1}$, Wioleta Stolarek ${ }^{1}$, \\ Zofia Grąbczewska ${ }^{1}$, Piotr Michalski ${ }^{1}$, Piotr Niezgoda ${ }^{1}$, Stanisław Bartuś ${ }^{2}$, \\ Andrzej Budaj ${ }^{3}$, Mariusz Dąbrowski ${ }^{4}$, Jarosław Drożd ${ }^{5}$, Ryszard Gellert ${ }^{6}$, \\ Miłosz J. Jaguszewski ${ }^{7}$, Piotr Jankowski ${ }^{8}$, Jacek Legutko ${ }^{9}$, Maciej Lesiak ${ }^{10}$, \\ Przemysław Leszek ${ }^{11}$, Jolanta Małyszko ${ }^{12}$, Przemysław Mitkowski ${ }^{10}$, \\ Jadwiga Nessler $^{13}$, Krzysztof Pawlaczyk ${ }^{14}$, Jolanta Siller-Matula ${ }^{15,16}$, \\ Tomasz Stompór ${ }^{17}$, Bogumił Wolnik ${ }^{18}$, Eliano Pio Navarese ${ }^{1}$ \\ ${ }^{1}$ Collegium Medicum, Nicolaus Copernicus University, Bydgoszcz, Poland \\ ${ }^{2} 2^{\text {nd }}$ Department of Cardiology, Collegium Medicum, Jagiellonian University, Krakow, Poland \\ ${ }^{3}$ Center of Postgraduate Medical Education, Department of Cardiology, \\ Grochowski Hospital, Warsaw, Poland \\ ${ }^{4}$ College of Medical Sciences, University of Rzeszow, Poland \\ ${ }^{5} 2^{\text {nd }}$ Department of Cardiology, Chair of Cardiology, Cardiac Surgery and Vascular Diseases, \\ Medical University of Lodz, Poland \\ ${ }^{6}$ Department of Nephrology and Internal Medicine, \\ Center of Postgraduate Medical Education, Warsaw, Poland \\ ${ }^{7} 1^{\text {st }}$ Department of Cardiology, Medical University of Gdansk, Poland \\ ${ }^{8} 1^{\text {st }}$ Department of Cardiology, Collegium Medicum, Jagiellonian University, Krakow, Poland
} ${ }^{9}$ Department of Interventional Cardiology, Institute of Cardiology, Jagiellonian University Medical College, John Paul II Hospital in Krakow, Poland

${ }^{10}$ Department of Cardiology, Poznan University of Medical Sciences, Poznan, Poland

${ }^{11}$ Department of Heart Failure and Transplantology, National Institute of Cardiology, Warsaw, Poland

${ }^{12}$ Department of Nephrology, Dialysis and Internal Medicine, Medical University of Warsaw, Poland

${ }^{13}$ Department of Coronary Artery Disease and Heart Failure, Institute of Cardiology,

Jagiellonian University Medical College, Krakow, Poland

${ }^{14}$ Department of Nephrology, Transplantology and Internal Diseases,

Poznan University of Medical Sciences, Poznan, Poland

${ }^{15}$ Department of Experimental and Clinical Pharmacology, Medical University of Warsaw, Poland

${ }^{16}$ Department of Cardiology, Medical University of Vienna, Austria

${ }^{17}$ Chair of Internal Medicine Department of Nephrology, Hypertension and Internal Medicine,

Medical Faculty, University of Warmia and Mazury in Olsztyn, Poland

${ }^{18}$ Department of Hypertension and Diabetology, Faculty of Medicine,

Medical University of Gdansk, Poland

Address for correspondence: Dr. Piotr Niezgoda, Department of Cardiology and Internal Medicine, Collegium Medicum in Bydgoszcz, ul. Skłodowskiej-Curie 9, 85-094 Bydgoszcz, Poland, tel: +48 52585 4023, fax (optional): +48 525854024 , e-mail: piotr.niezgoda1986@gmail.com

Received: 19.07.2021 Accepted: 6.08.2021 Early publication date: 25.10.2021

This article is available in open access under Creative Common Attribution-Non-Commercial-No Derivatives 4.0 International (CC BY-NC-ND 4.0) license, allowing to download articles and share them with others as long as they credit the authors and the publisher, but without permission to change them in any way or use them commercially. 


\begin{abstract}
Several mechanisms have been suggested to explain positive cardiovascular effects observed in studies with sodium-glucose co-transporter 2 (SGLT2) inhibitors. The reduction in glucose reabsorption in proximal tubuli induced by SGLT2 inhibitors increases urinary glucose and sodium excretion resulting in increased osmotic diuresis and consequently in decreased plasma volume, followed by reduced preload. In addition, the hemodynamic effects of SGLT2 inhibition were observed in both hyper and euglycemic patients. Due to the complex and multidirectional effects induced by SGLT2 inhibitors, this originally antidiabetic group of drugs has been successfully used to treat patients with heart failure as well as for subjects with chronic kidney disease. Moreover, their therapeutic potential seems to be even broader than the indications studied to date. (Cardiol J 2023; 30, 1: 143-149)
\end{abstract}

Key words: sodium-glucose co-transporters (SGLTs), dapagliflozin, empagliflozin, heart failure, mechanisms

\section{Sodium-glucose co-transporters}

Sodium-glucose co-transporters (SGLTs) are a family of transmembrane proteins characterized by a shared transportation mechanism, in which extracellular sodium binding triggers a gate to open and trap glucose from outside the cell. Subsequently, the transporter flips releasing glucose and sodium into the cytoplasm. At the end of the process the protein returns to its initial conformation. SGLT1 and SGLT2 are the two most known SGLTs [1].

The SGLT2 is mainly expressed in the kidneys on the epithelial cells in the proximal convoluted tubule. In spite of low affinity for glucose, the SGLT2-mediated renal reabsorption demonstrates a very high capacity [2]. The reabsorption of glucose can be characterized as sodium-dependent with ratios of $1: 1$ and 2:1 for SGLT2 and SGLT1, respectively [1]. With only a fraction of tubular glucose reabsorption being SGLT1-mediated, the majority of the process, approximately $90 \%$, is handled by SGLT2. The kidney capacity of reabsorbing of filtered glucose represents an extremely efficient mechanism of energy conservation. In addition to the kidney, the SGLT2 expression was demonstrated in the brain, liver, thyroid, muscles, and heart, while the SGLT1 expression was found in the intestine, trachea, kidney, heart, brain, testis, and prostate [1].

\section{Sodium-glucose co-transporters inhibition}

The first description of phlorizin's ability to block renal glucose reabsorption in humans was published in 1933 [3]. Phlorizin is a glucoside of phloretin (a bicyclic flavonoid), which can mainly be found in apples, specifically in unripe fruits and root bark, as well as in smaller quantities in strawberries. Phlorizin, a competitive nonspecific SGLT inhibitor, competes for binding with D-glucose. It reduces both postprandial and fasting glycemia as shown in experimental animal model studies [4]. Due to these properties, orally active selective inhibitors of SGLT2 have been applied as antidiabetic agents. The glucose reabsorption limited by SGLT2 inhibitors (SGLT2i) is characterized by the rapid onset of glycosuria (1-2 h), the dose-dependent offset of action in the range of $24-48 \mathrm{~h}$ and the glycemia-dependent entity of glycosuria. Inhibition of SGLT2 lowers the renal glucose threshold into the euglycemic range. In humans, glucose reabsorption inhibition can maximally reach between $30 \%$ and $50 \%$ [5]. Glycosuria induced by SGLT2i in diabetic patients results in further metabolic changes. The decrease in fasting and postprandial plasma glucose concentrations is caused by a partial drainage of glucose from the extracellular space. Plasma insulin concentration and the secretion rate declines while plasma glucagon rises following the glycemia changes. A decreased insulin-to-glucagon ratio alters hepatic glucose metabolism, inducing endogenous fasting gluconeogenesis and attenuating its postprandial suppression [1]. Lower insulin levels reduce the lipolysis inhibition and cause an excess of free fatty acids to reach the tissues via the bloodstream. The extra free fatty acids delivery, together with the insulin-to-glucagon ratio reduction translates into ketogenesis $[5,6]$. Normally, the excess of free fatty acids would lead to insulin resistance by competing with glucose utilization. However, on treatment with SGLT2i it is paradoxically associated with improved insulin sensitivity [5, 7, 8]. A schematic presentation of SGLT2 inhibitors' mechanism of action is shown in the Central illustration. 


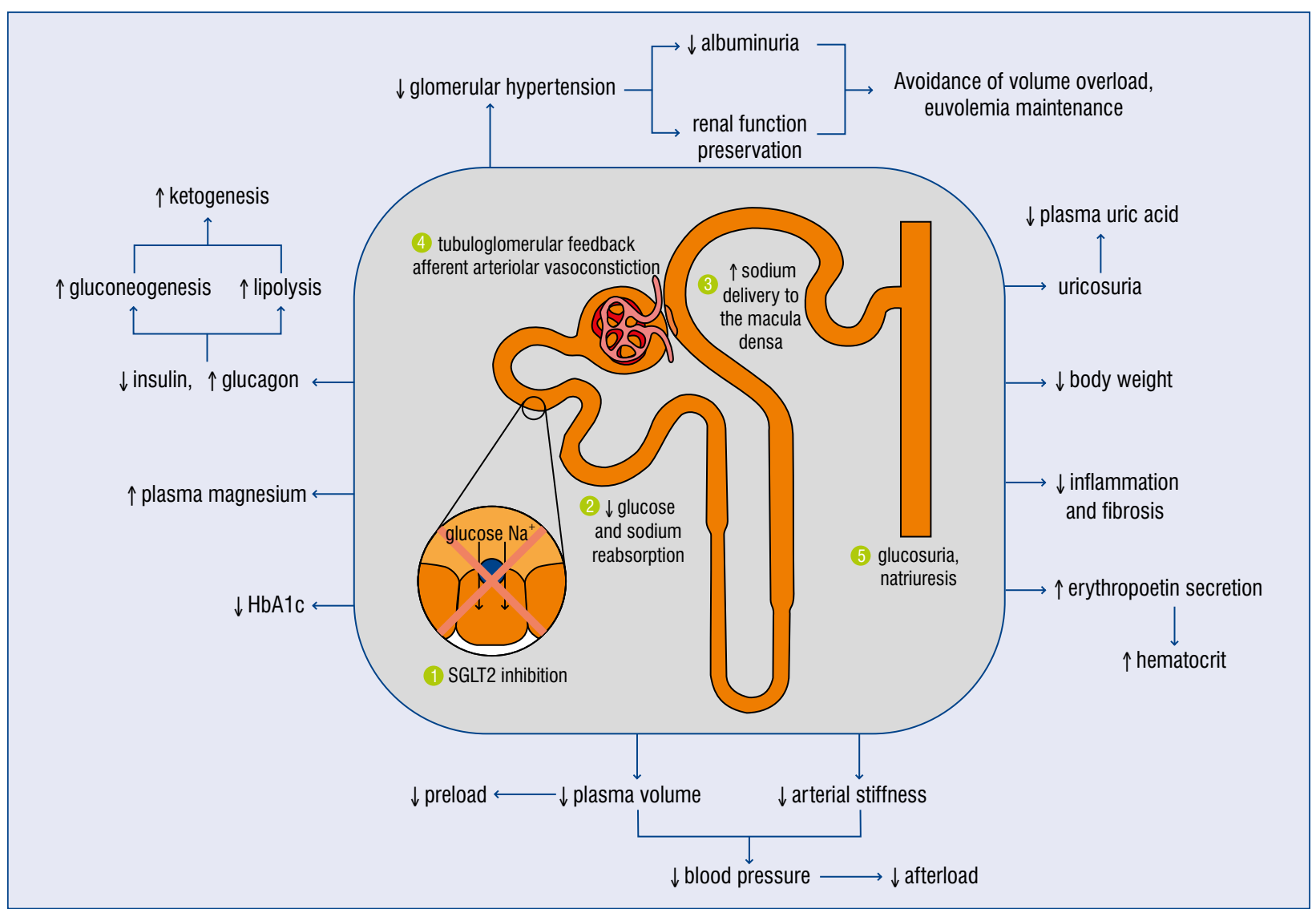

Central illustration. Mechanisms of action of sodium-glucose co-transporter 2 (SGLT2) inhibitors.

\section{Clinical effects of sodium-glucose co-transporters inhibition}

The loss of glucose on SGLT inhibitors channels into a substantial, between $250 \mathrm{kcal} /$ day and $450 \mathrm{kcal} /$ day, whole-body deficit of energy. However, based on this negative calorie balance, the weight loss observed in clinical trials was far less than expected, which might be explained by an increased caloric intake [9]. Countering this increase, through e.g., dietary counseling or pharmacological appetite quenching, could potentially strengthen the weight loss induced by SGLT2i $[6,9]$. Complex metabolic changes induced by SGLT2i, including delayed clearance of low-density lipoprotein cholesterol (LDL-C) from the circulation along with increased plasma lipoprotein lipase activity, lead to increase of LDL-C and decrease of triglycerides plasma levels [8].

Additional properties of SGLT2i include a dose-dependent reduction of serum uric acid levels [10] as well as a reduction of urinary albumin excretion in type 2 diabetes patients with prevalent micro- or macroalbuminuria [11].
Several mechanisms have been suggested to explain positive cardiovascular effects observed in studies with SGLT2i [1, 6, 12-14]. Heart failure (HF) is a condition characterized by excessive accumulation of fluid in the vascular compartment as well as in the interstitial space. Still, many patients suffering from HF experience arterial underfilling due to a low cardiac output, which sometimes can be intensified by conventional treatment with diuretics. The reduction in glucose reabsorption in proximal tubuli induced by SGLT2i increases urinary glucose and sodium excretion resulting in increased osmotic diuresis and consequently in decreased plasma volume, followed by a reduced preload $[6,15]$.

Concomitant lowering of arterial stiffness and blood pressure leads to the afterload reduction $[6,15]$. Sympathetic activation as well as decreased arterial filling, organ perfusion and blood pressure constitute some of the potential negative effects of excessively reducing blood volume [16]. However, heart rate has not been found to increase on treatment with SGLT2i despite the consistent fall in arterial blood pressure in patients with type 2 dia- 
betes, indicating that autonomic balance shifts toward the parasympathetic branch [1]. According to experimental studies, a more significant reduction of interstitial fluid volume than that of blood volume can be observed with SGLT2i. Hence, they could serve as better congestion control agents without diminishing arterial filling and tissue perfusion in contrast to conventional diuretic treatment [16]. The beneficial effect of SGLT2i on left ventricular (LV) systolic (an increase of LV ejection fraction) and diastolic (a decrease of LV filling pressure) function was shown in patients with HF. Moreover, a significant decrease in LV mass index, left atrial volume index was observed. These findings were associated with a significant decrease in plasma B-type natriuretic peptide concentration only in patients with a baseline value $\geq 100 \mathrm{pg} / \mathrm{mL}$ [17].

The hemodynamic effects of SGLT2 inhibition were observed in both hyper and euglycemic patients $[1,5]$. Regardless of these favorable changes a decrease of hemoglobin $\mathrm{A} 1 \mathrm{c}$ (HbA1c) concentration, body weight, and blood pressure, as well as an increase in hematocrit, hemoglobin and albumin concentration, were noted $[1,5,12,18-20]$.

It has been proposed that the SGLT2 inhibition driven effects can be explained by the restoration of both the anabolic and catabolic states cycling and the housekeeping processes of the cells, which are facilitated by caloric offloading through glucosuria. This fasting-like effect, not seen with other antihyperglycemic drugs, triggers nutrient deprivation pathways, including stimulation of gluconeogenesis and ketogenesis to promote cellular homeostasis [21-23]. The gluconeogenesis and ketogenesis are regulated by endogenous nutrient deprivation sensors silent information regulator $\mathrm{T} 1$, proliferator-activated receptor gamma coactivator 1 -alpha and fibroblast growth factor 21 , which are known to exert cardioprotective effects in experimental models [23]. The aforementioned benefit occurs to be mediated by alleviating oxidative stress and promoting autophagy. This degradative pathway plays a crucial role in lysosome-dependent disposing of dysfunctional cellular components that constitute a significant cause of cell damage [13, 22, 23].

On the other hand, clinical trials have not shown a reduction in HF events related to metformin stimulation of the adenosine monophosphate-activated protein kinase, a known sensor for nutrient deprivation that does not induce ketogenesis. Therefore, the main focus of the positive cardiovascular and renal effects of SGLT2 inhibition is the boost in ketone bodies production as a more potent fuel source for mitochondria compared to free fatty acids $[1,5,12,13,23,24]$. The rapid-onset renal and cardiovascular benefits demonstrated in the trials' outcome remain consistent with this very elegant metabolic theory [13, 18, 19, 25].

It has also been postulated that SGLT2i directly inhibit $\mathrm{Na}+/ \mathrm{H}+$ exchanger (NHE) 1 , leading to reduced sodium and calcium cytoplasmic levels and to increase in mitochondrial calcium levels, thus restoring mitochondrial function and stimulating energy production [26, 27].

Solini et al. [28] demonstrated the influence of dapagliflozin on the expression of miRNAs related to HF pathophysiology: upregulation of miR30e-5p and downregulation of miR199a-3p. Moreover, SGLT2 inhibition preserves renal vasodilating capacity. The aforementioned effects operate independently of the diuretic and blood pressurelowering properties of dapagliflozin [29]. They also showed a significant dapagliflozin-induced rise in magnesium concentrations, which are known to be inversely associated with risk for coronary heart disease and HF deaths. Therefore, this might contribute to the cardiovascular protection exerted by SGLT2i [28, 30].

Administration of SGLT2i is associated with the acute decrease in creatinine clearance related to resetting macula densa tubuloglomerular feedback. Inhibition of proximal sodium absorption delivers an excess of sodium to the macula densa, which acts as a trigger releasing vasoconstrictive molecules. This mechanism results in vasoconstriction of the glomerular afferent arterioles reduces glomerular filtration rate and protects against the harmful impact of blood pressure increases and fluctuations on the glomerular capillaries located downstream [31]. Nevertheless, the renoprotective effects that SGLT2i exert cannot be easily explained only by their actions to lower blood glucose, blood pressure, or glomerular filtration pressures.

In the cardiovascular outcome trials increase in hematocrit was observed, but the mechanism of this phenomenon was not clearly explained [18, 19]. In the EMPA-HEART study, empagliflozin use was associated with an increase in erythropoietin level. Several mechanisms at the kidney level may be responsible for this effect [32]. The rise in hematocrit suggests that SGLT2i influence hypoxiainducible factors (HIFs; specifically, HIF- $1 \alpha$ and HIF- $2 \alpha$ ). This property could be the underlying reason for their ability to prevent nephropathy from progressing [33]. Defective nutrient deprivation signaling, oxidative and endoplasmic reticulum stress as well as renal hypoxia are all features of type 2 diabetes. These conditions tend to cause 
Table 1. Multidirectional mechanisms of sodium-glucose co-transporter 2 (SGLT2) inhibitors activity in various clinical indications.

\begin{tabular}{|c|c|c|c|}
\hline Clinical setting & Heart failure & Type 2 diabetes & Chronic kidney disease \\
\hline $\begin{array}{c}\text { Pathophysiological } \\
\text { effect }\end{array}$ & $\begin{array}{l}\text { Increased urinary sodium } \\
\text { excretion, increased } \\
\text { osmotic diuresis, decreased } \\
\text { plasma and interstitial fluid } \\
\text { volume, decreased preload, } \\
\text { decreased arterial stiffness, } \\
\text { decreased afterload, } \\
\text { improved autonomic } \\
\text { balance, decreased inflam- } \\
\text { mation, decreased plasma } \\
\text { B-type natriuretic peptide, } \\
\text { increased plasma Mg }\end{array}$ & $\begin{array}{c}\text { Decreased glucose } \\
\text { reabsorption, increased } \\
\text { insulin sensitivity, decreased } \\
\text { HbA1c, decreased inflam- } \\
\text { mation, whole body energy } \\
\text { deficit, weight loss }\end{array}$ & $\begin{array}{l}\text { Decreased urinary albumin } \\
\text { excretion, decreased plasma } \\
\text { B-type natriuretic peptide, } \\
\text { decreased inflammation, } \\
\text { preserved renal vasodilating } \\
\text { capacity, reduced glomerular } \\
\text { hypertension, improved } \\
\text { renal function, } \\
\text { attenuated diabetes-induced } \\
\text { tubulointerstitial fibrosis }\end{array}$ \\
\hline
\end{tabular}

a shift in HIF- $1 \alpha / \mathrm{HIF}-2 \alpha$ activities ratio through simultaneous HIF- $1 \alpha$ activation and HIF- $2 \alpha$ suppression. The resulting balance change stimulates profibrotic and proinflammatory pathways in both tubular and glomerular cells [33]. Several small studies with SGLT2i confirmed a slight reduction in serum inflammatory markers: high sensitivity C-reactive protein, tumor necrosis factor-alpha, interleukin-6, and interferon-gamma, which was summarized by Bonnet and Sheen [29]. Moreover, SGLT2 inhibition with dapagliflozin was shown to slow down the progression of diabetes-associated renal tubulointerstitial fibrosis by improving hyperglycemia-induced activation of the signal transducer and activator of transcription-1/transforming growth factor-beta 1 pathway [34]. Thus, reduction of inflammation can be considered as another mechanism explaining the beneficial effect of SGLT2i at the kidney and myocardium levels.

The summary of mechanisms of SGLT2i in various clinical settings is presented in Table 1.

\section{Sodium-glucose co-transporters inhibitors}

Several SGLT2i have been developed, including canagliflozin, dapagliflozin, empagliflozin, ertugliflozin, ipragliflozin, luseogliflozin, remogliflozin, sergliflozin, sotagliflozin, and tofogliflozin; however, only a few of them have been tested in large-scale, long-term, randomized clinical trials. Only dapagliflozin and empagliflozin were evaluated in patients with $\mathrm{HF}$ regardless of a diabetes diagnosis [35-37]. Both these agents have shown high efficacy in reducing the primary outcome defined as a composite of worsening HF or cardiovascular death. Cardiovascular mortality and all-cause mortality were significantly decreased regardless of a diabetes diagnosis only with dapagliflozin [35], while in patients with type 2 diabetes, only with empagliflozin [12]. Treatment with empagliflozin and dapagliflozin was safe and well tolerated, resulting in excellent adherence to medication a key factor in the long-term treatment of patients with chronic diseases [38-49].

Based on the results of clinical trials, McMurray and Packer [50] proposed a new algorithm for the sequencing of foundational treatments in $\mathrm{HF}$ patients with reduced ejection fraction. The 3-step algorithm should be applied in a patient in whom clinical euvolemia has been achieved with diuretics. The treatment should be started with simultaneous initiation of treatment with a beta-blocker and an SGLT2i (step 1); the addition of sacubitril/ /valsartan (step 2); and the subsequent addition of an mineralocorticoid receptor antagonists (step 3). The proposed approach is expected to maximize the likelihood that highly effective therapies will be implemented in a manner that rapidly prevents deaths and hospitalizations and enhances the tolerability of concurrently or subsequently administered treatments [50].

\section{Conclusions}

Due to the complex and multidirectional mechanism of action of SGLT2i, this originally antidiabetic group of drugs has been successfully used to treat patients with $\mathrm{HF}$ and subjects with chronic kidney disease. Moreover, their therapeutic potential seems to be even wider than the indications studied so far.

Conflict of interest: Jacek Kubica, Aldona Kubica, Ryszard Gellert, Maciej Lesiak: personal fees from 
AstraZeneca; Andrzej Budaj, Jarosław Drożdż: personal fees and non-financial support from AstraZeneca; Tomasz Stompór: personal fees from AstraZeneca and Mundipharma, conduction of clinical trials sponsored by AstraZeneca; Piotr Jankowski: personal fees and travel grants from Boehringer-Ingelheim. All the remaining authors declare no conflict of interest regarding publication of this manuscript.

\section{References}

1. Ferrannini E. Sodium-Glucose co-transporters and their inhibition: clinical physiology. Cell Metab. 2017; 26(1): 27-38, doi: 10.1016/j.cmet.2017.04.011, indexed in Pubmed: 28506519.

2. Gallo LA, Wright EM, Vallon V. Probing SGLT2 as a therapeutic target for diabetes: basic physiology and consequences. Diab Vasc Dis Res. 2015; 12(2): 78-89, doi: 10.1177/1479164114561992, indexed in Pubmed: 25616707.

3. Chasis H, Jolliffe N, Smith HW. The action of phlorizin on the excretion of glucose, xylose, sucrose, creatinine and urea by man. J Clin Invest. 1933; 12(6): 1083-1090, doi: 10.1172/JCI100559, indexed in Pubmed: 16694183.

4. Wright EM, Loo DDF, Hirayama BA. Biology of human sodium glucose transporters. Physiol Rev. 2011; 91(2): 733-794, doi: 10.1152/physrev.00055.2009, indexed in Pubmed: 21527736.

5. Ferrannini E, Muscelli E, Frascerra S, et al. Metabolic response to sodium-glucose cotransporter 2 inhibition in type 2 diabetic patients. J Clin Invest. 2014; 124(2): 499-508, doi: 10.1172/ JCI72227, indexed in Pubmed: 24463454.

6. Lytvyn Y, Bjornstad P, Udell JA, et al. Sodium glucose cotransporter-2 inhibition in heart failure: potential mechanisms, clinical applications, and summary of clinical trials. Circulation. 2017; 136(17): 1643-1658, doi: 10.1161/CIRCULATIONAHA.117.030012, indexed in Pubmed: 29061576.

7. Merovci A, Abdul-Ghani M, Mari A, et al. Effect of dapagliflozin with and without acipimox on insulin sensitivity and insulin secretion in T2DM males. J Clin Endocrinol Metab. 2016; 101(3): 1249-1256, doi: 10.1210/jc.2015-2597, indexed in Pubmed: 26765576.

8. Basu D, Huggins LA, Scerbo D, et al. Mechanism of increased LDL (low-density lipoprotein) and decreased triglycerides with SGLT2 (sodium-glucose cotransporter 2) inhibition. Arterioscler Thromb Vasc Biol. 2018; 38(9): 2207-2216, doi: 10.1161/ATVBAHA.118.311339, indexed in Pubmed: 30354257.

9. Ferrannini G, Hach T, Crowe S, et al. Energy balance after sodium-glucose cotransporter 2 inhibition. Diabetes Care. 2015; 38(9): 1730-1735, doi: 10.2337/dc15-0355, indexed in Pubmed: 26180105.

10. DeFronzo RA, Cooke CR, Andres R, et al. The effect of insulin on renal handling of sodium, potassium, calcium, and phosphate in man. J Clin Invest. 1975; 55(4): 845-855, doi: 10.1172/JCI107996, indexed in Pubmed: 1120786.

11. Cherney D, Lund SS, Perkins BA, et al. The effect of sodium glucose cotransporter 2 inhibition with empagliflozin on microalbuminuria and macroalbuminuria in patients with type 2 diabetes. Diabetologia. 2016; 59(9): 1860-1870, doi: 10.1007/ s00125-016-4008-2, indexed in Pubmed: 27316632.

12. Inzucchi SE, Zinman B, Fitchett D, et al. How does empagliflozin reduce cardiovascular mortality? Insights from a mediation analysis of the EMPA-REG OUTCOME trial. Diabetes Care. 2018; 41(2): 356-363, doi: 10.2337/dc17-1096, indexed in Pubmed: 29203583.

13. Esterline RL, Vaag A, Oscarsson J, et al. Mechanisms in endocrinology: SGLT2 inhibitors: clinical benefits by restoration of normal diurnal metabolism? Eur J Endocrinol. 2018; 178(4): R113-R125, doi: 10.1530/EJE-17-0832, indexed in Pubmed: 29371333.

14. Jardine MJ, Mahaffey KW, Neal B, et al. CREDENCE study investigators. The Canagliflozin and Renal Endpoints in Diabetes with Established Nephropathy Clinical Evaluation (CREDENCE) Study Rationale, Design, and Baseline Characteristics. Am J Nephrol. 2017 [Epub ahead of print]; 46(6): 462-472, doi: 10.1159/000484633, indexed in Pubmed: 29253846.

15. Dekkers CCJ, Sjöström CD, Greasley PJ, et al. Effects of the sodium-glucose co-transporter-2 inhibitor dapagliflozin on estimated plasma volume in patients with type 2 diabetes. Diabetes Obes Metab. 2019; 21(12): 2667-2673, doi: 10.1111/dom.13855, indexed in Pubmed: 31407856.

16. Hallow KM, Helmlinger G, Greasley PJ, et al. Why do SGLT2 inhibitors reduce heart failure hospitalization? A differential volume regulation hypothesis. Diabetes Obes Metab. 2018; 20(3): 479-487, doi: 10.1111/dom.13126, indexed in Pubmed: 29024278.

17. Soga F, Tanaka H, Tatsumi K, et al. Impact of dapagliflozin on left ventricular diastolic function of patients with type 2 diabetic mellitus with chronic heart failure. Cardiovasc Diabetol. 2018; 17(1): 132, doi: 10.1186/s12933-018-0775-z, indexed in Pubmed: 30296931.

18. Zinman B, Wanner C, Lachin JM, et al. EMPA-REG OUTCOME Investigators. Empagliflozin, Cardiovascular Outcomes, and Mortality in Type 2 Diabetes. N Engl J Med. 2015; 373(22): 2117-2128, doi: 10.1056/NEJMoa1504720, indexed in Pubmed: 26378978.

19. Neal B, Perkovic V, Mahaffey KW, et al. CANVAS Program Collaborative Group. Canagliflozin and cardiovascular and renal events in type 2 diabetes. N Engl J Med. 2017; 377(7): 644-657, doi: 10.1056/NEJMoa1611925, indexed in Pubmed: 28605608.

20. Phrommintikul A, Wongcharoen W, Kumfu S, et al. Effects of dapagliflozin vs vildagliptin on cardiometabolic parameters in diabetic patients with coronary artery disease: a randomised study. Br J Clin Pharmacol. 2019; 85(6): 1337-1347, doi: 10.1111/ bcp.13903, indexed in Pubmed: 30767253.

21. Packer M. Molecular, cellular, and clinical evidence that sodiumglucose cotransporter 2 inhibitors act as neurohormonal antagonists when used for the treatment of chronic heart failure. J Am Heart Assoc. 2020; 9(16): e016270, doi: 10.1161/ JAHA.120.016270, indexed in Pubmed: 32791029.

22. Luo G, Jian Z, Zhu Y, et al. Sirt1 promotes autophagy and inhibits apoptosis to protect cardiomyocytes from hypoxic stress. Int J Mol Med. 2019; 43(5): 2033-2043, doi: 10.3892/ijmm.2019.4125, indexed in Pubmed: 30864731.

23. Packer M. Cardioprotective effects of sirtuin-1 and its downstream effectors: potential role in mediating the heart failure benefits of SGLT2 (sodium-glucose cotransporter 2) inhibitors. Circ Heart Fail. 2020; 13(9): e007197, doi: 10.1161/CIRCHEARTFAILURE.120.007197, indexed in Pubmed: 32894987.

24. Neubauer S. The failing heart--an engine out of fuel. N Engl J Med. 2007; 356(11): 1140-1151, doi: 10.1056/NEJMra063052, indexed in Pubmed: 17360992.

25. Rådholm K, Figtree G, Perkovic V, et al. Canagliflozin and heart failure in type 2 diabetes mellitus. Results From the CANVAS 
Program. Circulation. 2018; 138(5): 458-468, doi: 10.1161/circulationaha.118.034222, indexed in Pubmed: 29526832.

26. Zelniker TA, Braunwald E. Mechanisms of cardiorenal effects of sodium-glucose cotransporter 2 inhibitors: JACC state-of-the-art review. J Am Coll Cardiol. 2020; 75(4): 422-434, doi: 10.1016/j. jacc.2019.11.031, indexed in Pubmed: 32000955.

27. Baartscheer A, Schumacher CA, Wüst RCI, et al. Empagliflozin decreases myocardial cytoplasmic Na through inhibition of the cardiac $\mathrm{Na} / \mathrm{H}$ exchanger in rats and rabbits. Diabetologia. 2017; 60(3): 568-573, doi: 10.1007/s00125-016-4134-x, indexed in Pubmed: 27752710 .

28. Solini A, Seghieri M, Giannini L, et al. The effects of dapagliflozin on systemic and renal vascular function display an epigenetic signature. J Clin Endocrinol Metab. 2019; 104(10): 4253-4263, doi: 10.1210/jc.2019-00706, indexed in Pubmed: 31162549.

29. Bonnet F, Scheen AJ. Effects of SGLT2 inhibitors on systemic and tissue low-grade inflammation: The potential contribution to diabetes complications and cardiovascular disease. Diabetes Metab. 2018; 44(6): 457-464, doi: 10.1016/j.diabet.2018.09.005, indexed in Pubmed: 30266577.

30. Kieboom BCT, Niemeijer MN, Leening MJG, et al. Serum magnesium and the risk of death from coronary heart disease and sudden cardiac death. J Am Heart Assoc. 2016; 5(1), doi: 10.1161/ JAHA.115.002707, indexed in Pubmed: 26802105.

31. Carlström M, Wilcox CS, Arendshorst WJ. Renal autoregulation in health and disease. Physiol Rev. 2015; 95(2): 405-511, doi: 10.1152/physrev.00042.2012, indexed in Pubmed: 25834230.

32. Mazer CD, Hare GMT, Connelly PW, et al. Effect of empagliflozin on erythropoietin levels, iron stores, and red blood cell morphology in patients with type 2 diabetes mellitus and coronary artery disease. Circulation. 2020; 141(8): 704-707, doi: 10.1161/CIRCULATIONAHA.119.044235, indexed in Pubmed: 31707794.

33. Packer M. Mechanisms leading to differential hypoxia-inducible factor signaling in the diabetic kidney: modulation by SGLT2 inhibitors and hypoxia mimetics. Am J Kidney Dis. 2021; 77(2): 280-286, doi: 10.1053/j.ajkd.2020.04.016, indexed in Pubmed: 32711072 .

34. Huang F, Zhao Y, Wang Q, et al. Dapagliflozin attenuates renal tubulointerstitial fibrosis associated with type 1 diabetes by regulating stat1/tgf $\beta 1$ signaling. Front Endocrinol. 2019; 10, doi: 10.3389/fendo.2019.00441.

35. McMurray JJV, Solomon SD, Inzucchi SE, et al. DAPA-HF Trial Committees and Investigators. Dapagliflozin in patients with heart failure and reduced ejection fraction. N Engl J Med. 2019; 381(21): 1995-2008, doi: 10.1056/NEJMoa1911303, indexed in Pubmed: 31535829.

36. Zannad F, Ferreira JP, Pocock SJ, et al. SGLT2 inhibitors in patients with heart failure with reduced ejection fraction: a meta-analysis of the EMPEROR-Reduced and DAPA-HF trials. Lancet. 2020; 396(10254): 819-829, doi: 10.1016/S01406736(20)31824-9, indexed in Pubmed: 32877652.

37. Packer M, Anker SD, Butler J, et al. EMPEROR-Reduced Trial Investigators. Cardiovascular and renal outcomes with empagliflozin in heart failure. N Engl J Med. 2020; 383(15): 1413-1424, doi: 10.1056/NEJMoa2022190, indexed in Pubmed: 32865377.

38. Kubica A, Kosobucka A, Fabiszak T, et al. Assessment of adherence to medication in patients after myocardial infarction treated with percutaneous coronary intervention. Is there a place for newself-reported questionnaires? Curr Med Res Opin. 2019; 35(2): 341-349, doi: 10.1080/03007995.2018.1510385, indexed in Pubmed: 30091642.

39. Kosobucka A, Michalski P, Pietrzykowski $Ł$, et al. Adherence to treatment assessed with the adherence in chronic diseases scale in patients after myocardial infarction. Patient Prefer Adherence. 2018; 12: 333-340, doi: 10.2147/PPA.S150435, indexed in Pubmed: 29551891.

40. Kubica A, Obońska K, Fabiszak T, et al. Adherence to antiplatelet treatment with P2Y12 receptor inhibitors. Is there anything we can do to improve it? A systematic review of randomized trials. Curr Med Res Opin. 2016; 32(8): 1441-1451, doi: 10.1080/03007995.2016.1182901, indexed in Pubmed: 27112628.

41. Kubica A, Kasprzak M, Obońska K, et al. Discrepancies in assessment of adherence to antiplatelet treatment after myocardial infarction. Pharmacology. 2015; 95(1-2): 50-58, doi: 10.1159/000371392, indexed in Pubmed: 25592409.

42. Kubica A, Kasprzak M, Siller-Matula J, et al. Time-related changes in determinants of antiplatelet effect of clopidogrel in patients after myocardial infarction. Eur J Pharmacol. 2014; 742: 47-54, doi: 10.1016/j.ejphar.2014.08.009, indexed in Pubmed: 25199965.

43. Pietrzykowski $€$, Kasprzak M, Michalski P, et al. Therapy discontinuation after myocardial infarction. J Clin Med. 2020; 9(12), doi: 10.3390/jcm9124109, indexed in Pubmed: 33352811.

44. Kubica A, Gruchała M, Jaguszewski M, et al. Adherence to treatment - a pivotal issue in long-term treatment of patients with cardiovascular diseases. An expert standpoint. Med Res J. 2018; 2(4): 123-127, doi: 10.5603/mrj.2017.0016.

45. Pietrzykowski $Ł$, Michalski P, Kosobucka A, et al. Medication adherence and its determinants in patients after myocardial infarction. Sci Rep. 2020; 10(1): 12028, doi: 10.1038/s41598-02068915-1, indexed in Pubmed: 32694522.

46. Kubica A, Adamski P, Bączkowska A, et al. The rationale for Multilevel Educational and Motivational Intervention in Patients after Myocardial Infarction (MEDMOTION) project is to support multicentre randomized clinical trial Evaluating Safety and Efficacy of Two Ticagrelor-based De-escalation Antiplatelet Strategies in Acute Coronary Syndrome (ELECTRA - SIRIO 2). Med Res J. 2020; 5(4): 244-249, doi: 10.5603/mrj.a2020.0043.

47. Kubica A. Self-reported questionnaires for a comprehensive assessment of patients after acute coronary syndrome. Med Res J. 2019; 4(2): 106-109, doi: 10.5603/mrj.a2019.0021.

48. Kosiborod MN, Jhund PS, Docherty KF, et al. Effects of dapagliflozin on symptoms, function, and quality of life in patients with heart failure and reduced ejection fraction: results from the DAPA-HF trial. Circulation. 2020; 141(2): 90-99, doi: 10.1161/ /CIRCULATIONAHA.119.044138, indexed in Pubmed: 31736335.

49. Butler J, Anker SD, Filippatos G, et al. EMPEROR-Reduced Trial Committees and Investigators. Empagliflozin and healthrelated quality of life outcomes in patients with heart failure with reduced ejection fraction: the EMPEROR-Reduced trial. Eur Heart J. 2021; 42(13): 1203-1212, doi: 10.1093/eurheartj/ ehaa1007, indexed in Pubmed: 33420498.

50. McMurray JJV, Packer M. How should we sequence the treatments for heart failure and a reduced ejection fraction?: A redefinition of evidence-based medicine. Circulation. 2021; 143(9): 875-877, doi: 10.1161/CIRCULATIONAHA.120.052926, indexed in Pubmed: 33378214. 